\title{
Effects of Formalin Toxicity among Preclinical I Medical Students, Adigrat University, Ethiopia
}

\section{Selemun Hagos ${ }^{1}$, Desta Gebeya ${ }^{1}$, Abraha Teklay²}

\author{
${ }^{1}$ Adigrat University, College of Medicine and Health Science, Department of Biomedical Sciences, Human \\ Anatomy Unit \\ ${ }^{2}$ Adigrat University College of Health Sciences, Department of Biomedical Sciences, Medical Physiology Unit
}

\begin{abstract}
Introduction: Formalin is a colorless gas with a pungent odor. Because of its germicidal efficacy it is widely used as a preservative for human cadaver in the anatomy laboratory. Acute exposure to formaldehyde results in irritation and burning of the mucous membranes of the nose, mouth, upper respiratory tract, depression, headache, nausea, coughing, irritation and burns to the skin and eye. Objective: The purpose of this study is to assess the effects of formalin toxicity among preclinical-I medical students in Adigrat University, Northern Ethiopia. Methodology: The study was conducted in anatomy laboratory, Adigrat University, Northern Ethiopia, which is located $894 \mathrm{Km}$ from the capital Addis Ababa. Cross sectional study design was utilized for this study. It was carried out on 96 preclinical-I medical students who were routinely exposed to formalin during cadaver dissection. Result: In this study highest investigation was responded as unpleasant smell which accounts for $84.4 \%$ students followed by tingling sensation of the nose that is $78.1 \%$. Effect of formalin on burning /watering of eye $67.7 \%$ and tiredness/dizziness $65.6 \%$ were also higher. Moreover, the data was showed that the effects of formalin accounts $17.7 \%$, $16.7 \%$ and $6.25 \%$, for abdominal pain, vomiting and fainting episodes, respectively. Conclusion: Anatomists, technicians and medical students who get regularly exposure to formalin at the dissection hall are at risk of formalin toxicity. Exposure to formalin vapour causes eye, nose, throat, and skin problems to the medical students. Therefore, this study reveals that the concerned bodies should take care of the side effects of formalin exposure.
\end{abstract}

Keywords: Formalin, Toxicity, Exposure, Preclinical, Dissection hall

\begin{abstract}
Introduction
Formalin is $37-40 \%$ aqueous solution that can dissolve in water. It is an organic carbon compound with pungent odor. Formalin is frequently used in hospitals, textiles, paper, resins and wood composites as fungicide, germicide, disinfectant and preservative $[1,2]$. Formalin can be mixed with alcohol, glycerine, phenol and ethanol for embalming and preserving of cadavers in the laboratory. It is also be used for fixation of tissue in the histology and histopathology laboratory. The Cadavers in the gross anatomy laboratories are preserved by formalin mixed solutions for long time. During dissection formalin vapours are emitted from the cadavers and affects the students and staffs of the anatomy laboratory [3].
\end{abstract}

The toxicity and harmful effect of formaldehyde is increased as the dose increased and when it becomes at room temperature due to its volatility property [4]. Formalin is an irritant compound which irritates the mucous membrane of the nose, throat and eyes.
Because of the toxic effect of formalin exposure, embalmers, anatomists, histology technicians and medical students are among the people who have high risk of effect of formalin toxicity [5]. The route of entrance for formaldehyde to the body is through inhalation and contact of skin. The quickly absorbed formalin from the nose and the upper part of lungs is quickly broken down [6].

The acute effects of formalin exposure are mainly as a result of its irritant properties. Acute high and long term low level of formalin exposures usually affect the eyes, upper and lower respiratory tract, skin and CNS. Formaldehyde is corrosive to the eyes and results in a burning sensation, lacrimation, conjunctivitis, corneal clouding and even loss of vision. In addition, Formaldehyde irritates the upper respiratory system and induces bronchial asthma, pulmonary edema, pneumonia and bronchospasm at an exposure above 50ppm [2].

This article is published under the terms of the Creative Commons Attribution License 4.0

Author(s) retain the copyright of this article. Publication rights with Alkhaer Publications.

Published at: http://www.ijsciences.com/pub/issue/2018-01/

DOI: 10.18483/ijSci.1479; Online ISSN: 2305-3925; Print ISSN: 2410-4477 
Anatomy instructors, laboratory technicians as well as medical students are routinely exposed to acute and chronic formalin vapour in the anatomy dissection room. These acute and chronic exposures of formalin adversely harm their health and evidenced by signs and symptoms. The signs and symptoms induced due to formalin exposure are among the crucial documentations of physiological changes that are important for further toxicological studies. Therefore, it is important to find out the toxicity level of formalin so as to create awareness of its toxicity. In this study toxic effect of acute exposure of formalin among preclinical I medical students exposed to formalin during their anatomy dissection session was evaluated.

\section{Objectives}

The objective of this study was to assess the effects of formalin toxicity among preclinical I medical students in the anatomy dissection room in Adigrat University, Northern Ethiopia.

\section{Methodology \\ Study area}

The study was conducted in anatomy dissection room, Adigrat University, Northern Ethiopia, which is located $894 \mathrm{Km}$ from the capital Addis Ababa.

\section{Study design}

Cross sectional study design was utilized for this study

\section{Study period}

Study was conducted on 2016/17 in Adigrat University Gross Anatomy dissection room.

\section{Study population}

The study population of this study were all preclinical I medical students in the academic year of 2016/17 in Adigrat University, Northern Ethiopia.

\section{Study participants}

Preclinical-I medical students were participate in this study who routinely exposed to formalin during cadaver dissection for two hours per day and two days a week regularly.

\section{Inclusion and exclusion criterion}

Students who are present during the data collection date, attending the laboratory session regularly and gave oral consent were included in the study. Whereas students with history of chronic cough and respiratory disease, asthma, allergic rhinitis, conjunctivitis, throat problems, dermatological system and epileptic disorder were excluded from the study.

\section{Sample size and sampling techniques}

Due to the limited number of students $(n=120)$ all of the participants were enrolled in the study sample size. Out of 120 students, 5 of them were not gave oral consent, 4 of them absent during data collection date, 8 of them were drop out and 7 of them had previous medical history. Only 96 preclinical I medical students were enrolled for this study.

\section{Method of data collection}

After participants were informed and oral consent was obtained from each participant; a selfadministered semi-structured questionnaire was given to each participant. The questionnaires were prepared based on signs and symptoms arising from exposure of formalin including effects on eyes, nose, throat, skin, concentration and dizziness.

\section{Data processing and analysis}

The collected data from 96 students was entered in to MS excel. Then percentage of signs and symptoms was done and presented in a table format.

\section{Results \\ Observation}

During cadaver dissection most of the students experienced tiredness, anxiety, headache, dizziness, difficulty of breathing and irritation of the eye and nose. In addition, some students also showed dryness of the lips, skin rash on hand and faint episode. However, fainting episode becomes reduced through time. During the dissection session the students feel the toxic effects but through time the students tend to adapt its toxicity.

\section{Sign and symptoms}

Table 1. Effects of formalin toxicity on preclinical -I medical student

\begin{tabular}{|c|l|c|c|}
\hline R/No. & Sign and symptoms & $\begin{array}{l}\text { Number of respondents } \\
\text { affected }\end{array}$ & $\begin{array}{l}\text { Percentage of respondents } \\
\text { affected }\end{array}$ \\
\hline 1. & Burning /watering of eye & 65 & $67.7 \%$ \\
\hline 2. & Irritation of upper respiratory tract & 52 & $54.2 \%$ \\
\hline 3. & Tingling sensation of the nose & 75 & $78.1 \%$ \\
\hline 4. & Skin problem & 18 & $18.8 \%$ \\
\hline 5. & Cough & 42 & $43.8 \%$ \\
\hline 6. & Lack of concentration & 43 & $44.8 \%$ \\
\hline 7. & Headache & 53 & $55.2 \%$ \\
\hline 8. & Difficulty in breathing & 60 & $62.5 \%$ \\
\hline
\end{tabular}




\begin{tabular}{|c|l|c|c|}
\hline 9. & Nausea & 50 & $52.1 \%$ \\
\hline 10. & Vomiting & 16 & $16.7 \%$ \\
\hline 11. & Abdominal pain & 17 & $17.7 \%$ \\
\hline 12. & Unpleasant smell & 81 & $84.4 \%$ \\
\hline 13. & Fainting episode & 6 & $6.25 \%$ \\
\hline 14. & Tiredness/dizziness & 63 & $65.6 \%$ \\
\hline
\end{tabular}

The results of toxic effects of formalin are presented on table 1 . In this study highest investigation was responded as unpleasant smell which accounts for $84.4 \%$ students followed by tingling sensation of the nose that is $78.1 \%$. Effect of formalin on burning /watering of eye $67.7 \%$ and Tiredness/dizziness $65.6 \%$ were also higher. In addition, from the study on toxic effect of formalin lower percentage of respondents was obtained as abdominal pain $17.7 \%$, vomiting $16.7 \%$ and fainting episode $6.25 \%$.

\section{Discussion}

Formalin is one of the most frequently used preservatives in most of the medical schools because of its antimicrobial efficacy. However, exposure to formalin causes coagulation necrosis, precipitation of protein, fixation of tissues and stabilizes the structural details of the cells [7]. Since it is easily available in the anatomy dissection room medical students and anatomy staffs are at a great disadvantage of formalin vapour toxicity. In this study unpleasant smell was reported in $84.4 \%$ of the respondents. This is in line with the study of Surajit and Pooja [8] unpleasant smell was found to be 94.68\% students. Another study conducted in Teheran medical school staff on occupational exposure of formaldehyde showed that most commonly reported complaints were unpleasant odor which accounts for $68 \%$ of the study participants [9].

The current study also showed $78.1 \%$ of students were found with tingling sensation of the nose. This is evidenced by the findings of the study conducted in India by Surajit and Pooja [8] and Teheran by Azira et al. [9]52.5\% and 52\% complaints were reported respectively. However, the difference between the results of the current studies and the studies conducted in India and Teheran might be due to the concentration of preparation of formalin for embalming and wetting solutions as well as the ventilation of the dissection room.

This study also showed that $67.7 \%$ of the respondents complain with burning/watering of eye. Similar results were also reported by Mrudula and Krishnaiah [10] and Patil et al. [11] which was $67 \%$ and $82 \%$ respectively. In addition, in this study it was also observed that most of the students experienced lacrimation and irritation of the eye. This observation was similar with finding of Patil et al. [11] who reported that toxic effect (irritation and watering of eyes) is first to be appear and most commonly seen in the study group, and it also sometimes prevents the students to come nearer to the dissecting cadaver. It was also observed that, most of the students attended in the cadaver dissection complained of running nose, headache and itching in skin. This observation also supported by shrivastava and Saxena [12] who, stated that most of the students engaged in cadaver dissection complained of head ache, nose lacrimation and itching skin.

Tiredness/dizziness $65.6 \%$, difficulty in breathing $62.5 \%$, irritation of upper respiratory tract $54.2 \%$, headache $55.2 \%$, nausea $52.1 \%$, cough $43.8 \%$, was also complained. Similar studies on formalin exposure conducted in Teheran and India was reported as cough (64\%), throat irritation (56\%), unusual tiredness $(31.92 \%)$, headache $(62.77 \%)$ and Nausea $(38.3 \%)$ subjects [8,9]. Different scholars also argued that, inhalation and exposure of formalin in large doses or exposure for long period of time in low doses results in irritation of lower respiratory tract and has posed risks of cancer particularly the upper respiratory tract $[11,13]$. Dry or sore throat, nasal itching and burning, and nasal congestion are evidenced as signs and symptoms of toxic effects of formaldehyde in upper respiratory system [2].

Lack of concentration (44.8\%), abdominal pain (17.7\%), Vomiting (16.7\%), and fainting episode $(6.25 \%)$ was also noted by the participants in the study. Similar study conducted in Koirala Institute of Health Sciences, department of anatomy dissection hall in 2015 revealed that $89.1 \%$ of the students were complained discomfort, nausea, vomiting and collapse on their first exposure to formalin due to its irritant nature of the formalin [14]. This difference might be due to the students adaptation to formalin as they stay longer. From the observation of this study it was observed that during the dissection session the students feel the toxic effects but through time the students tend to adapt the toxicity. Another study conducted in India on medical students routinely exposed to formalin vapour in 2015 disturbed concentration and post dissection vomiting was a problem in $27.67 \%$ and $4.26 \%$ students respectively [8].

In the current study $18.8 \%$ of studied students were experienced skin irritation/itching due to formalin exposure in the dissection hall. The study conducted by Koirala et al. [14] found that $82.2 \%$ of studied students experienced skin irritation/itching following 
their first exposure to Formalin. Moreover, itchiness of the skin of the hands was a symptom in $25.53 \%$ participants in the study conducted in India on medical students on the effect of formalin vapor emission in anatomy dissection hall [8]. This might be due to the fact that formalin solution exposure can cause allergic reactions, irritation of mucus membrane and skin rash. This is because formaldehyde is absorbed through intact skin and can cause severe irritation or allergic dermatitis. It can also cause white discoloration of the skin as well as burning, drying, cracking, blistering, and scaling of the skin. $[2,15]$.

Even though formaldehyde is toxic to different systems of the body, but because of its undoubted and consistent efficacy it remains the popular choice for tissue fixatives and cadaver preservatives. Reduced concentration of formaldehyde in the wetting fluid resulted in reduced vapor levels that reduce the toxic effects of formaldehyde.

\section{Conclusion}

Due to the effectiveness, low cost, and consistent results; formaldehyde is a popular choice of body preservative in the mortuary and anatomy dissection hall as tissue fixative despite of its toxic effects. The toxicity of formalin affects medical students who get regularly exposure to formalin during the anatomy laboratory session. Because of the wide use of formalin as preservative for cadavers in medical schools; anatomists, technicians and medical students who get regularly exposure to formalin at the dissection hall are at a great risk of formalin toxicity on various systems. Exposure or/and direct contact with formalin vapour causes eye, nose, throat and skin problems to the medical students. So it is important to take care of the side effects of formalin.

\section{Recommendations}

* The students and instructors as well as laboratory technicians should use protective equipments

* The gross anatomy dissection room should be well ventilated and regularly cleaned

* Formalin in the embalming solution and wetting solution should reduce as much as possible

* Think of other alternatives of formalin to reduce the side effects of formalin

* Further longitudinal studies with large sample size should be conducted

\section{Competing Interests}

Te authors declare no competing interests regarding the publication of this paper.

\section{Acknowledgment}

Authors are thankful to all those undergraduate medical students who participated in this study.

\section{References}

1. F.M. Onyije, and O.G. Avwioro, "Excruciating Effect of Formaldehyde Exposure to Students in Gross Anatomy Dissection Laboratory" Inter Journal occup environment med, Vol. 3 no.2, pp. 92-95, 2012.

2. D.S. Raja, and B. Sultana, "Potential Health Hazards for Students Exposed to Formaldehyde in the Gross Anatomy Laboratory", Journal Environmental Health, Vol 74, no. 6, pp. 36-40, 2002.

3. M. Takayanagi, M. Sakai, Y. Ishikawa, K. Murakami, A Kimura, and5 S. Kakuta, "Formaldehyde concentrations in the breathing zone of medical students during gross anatomy laboratory in Toho University", Kaibogaku Zasshi, Vol. 82, no.2, pp. 45- 51, 2007.

4. A. Songur, N. Akpolat, I. Kus, "The effects of the inhaled formaldehyde during the early postnatal period in the hippocampus of rats: a morphological and immunohistochemical study", Neur Res Com, Vol. 33, pp16 8-178, 2003.

5. D. Dixit, "Role of standardized embalming fluid in reducing the toxic effects of formaldehyde", Indian Journal Forensic Med Toxicol., Vol. 2, no.1, pp. 33-39, 2008.

6. Agency for Toxic Substances and Disease Registry (ATSDR), "Toxicological Profile for Formaldehyde", Public Health Service, pp165-167, 1999.

7. C .Surangama, M.R. Veeresh, "Formalin, a Rare Suicidal Poison" A Case Report. JDMS, Vol. 14, no. 4, pp 24-26, 2015 .

8. K. Surajit, and G. Pooja, "Study of the Toxic Effects of Formaldehyde Vapours within the Dissection Hall on the First Year Indian Medical Students", Inter Journal Anat Res., Vol. 3 no.2, pp1179-90, 2015.

9. M.R. Azari, A. Parisa, M.J. Jafari, Hamid, H. Vajihe, "Occupational Exposure of a Medical School Staff to Formaldehyde in Tehran" Tanaffos, Vol. 11, no.3, pp 36-41, 2012.

10. M. Krishnaiah, and C. Mrudula, "A Study on the Adverse Effects of Formaldehyde on the Staff of Anatomy Departments Working in Various Medical Colleges", Journal of Evolution of Medical and Dental Sciences, Vol. 1, pp559564, 2012.

11. G.V. Patil, Shishirkumar, Thejeshwari, D. Apoorva, J. Sharif, C. Sheshgiri, N.K. Sushant, "Physical reactions of formalin used as cadaver preservative on first year medical students" Journal Evidence Based Medicine and Healthcare, Vol.1 no. 5 pp 279-283, 2014

12. A. Shrivastava, and Y. Saxena, "Effect of Formalin Vapours on Pulmonary Functions of Medical Students in Anatomy Dissection Hall over a Period of One Year", Indian Journal Physiol Pharmacol., Vol. 57 no. 3, 2013.

13. K. Farah and P. Tripathi, "Acute effects of formalin on pulmonary functions in gross anatomy laboratory", Indian Journal Physiol Pharmacol. Vol. 53 no. 1, pp 93-96, 2009.

14. S. Koirala, S. Sandip, K. Laxman, P. Chudamani, and P. Depak, "Effect of Formalin among the medical and dental students attending regular laboratory session in dissecting hall, in Department of Human Anatomy, in B.P. Koirala Institute of Health Sciences", Euro. Journal Forensic Scie., Vol. 2 no. 2, pp1-4, 2015.

15. S. Takahashi, K. Tsuji, Fujii , F. Okazaki, T. Takigawa, A Ohtsuka, and K. Iwatsuki, "Prospective study of clinical symptoms and skin test reactions in medical students exposed to formaldehyde gas", Journal Dermatol., Vol. 34 no. 5, pp 283-289, 2007. 